\title{
Cloning and preliminary verification of telomere-associated sequences in upland cotton
}

\author{
Yuling Liu', Zhen Liu', Yangyang Wei', Yanjun Wang', Jiaran Shuang', \\ Renhai Peng'
}

I Anyang Institute of Technology, Anyang, Henan, 455000, China

Corresponding author: Renhai Peng (aydxprh@163.com)

Academic editor: V. Shneyer | Received 14 December 2019 | Accepted 4 March 2020 | Published 6 April 2020

http://zoobank.org/632FF29E-E61D-4AB1-89D3-172B79BB09EE

Citation: Liu Y, Liu Z, Wei Y, Wang Y, Shuang J, Peng R (2020) Cloning and preliminary verification of telomereassociated sequences in upland cotton. Comparative Cytogenetics 14(2): 183-195. https://doi.org/10.3897/ CompCytogen.v14i2.49391

\begin{abstract}
Telomeres are structures enriched in repetitive sequences at the end of chromosomes. In this study, using the telomere primer $\mathrm{AA}(\mathrm{CCCTAAA}){ }_{3} \mathrm{CCC}$ for the single primer PCR, two DNA sequences were obtained from Gossypium hirsutum (Linnaeus, 1753) accession (acc.) TM-1. Sequence analysis showed that the two obtained sequences were all rich in A/T base, which was consistent with the characteristic of the telomereassociated sequence (TAS). They were designated as GhTAS1 and GhTAS2 respectively. GhTAS1 is 489 bp long, with $57.6 \%$ of A/T, and GhTAS2 is 539 bp long, with $63.9 \%$ of A/T. Fluorescence in situ hybridization results showed that both of the cloned TASs were located at the ends of the partial chromosomes of G. hirsutum, with the strong signals, which further confirmed that GhTAS1 and GhTAS2 were telomereassociated sequences including highly tandemly repetitive sequences. Results of blast against the assembled genome of $G$. hirsutum showed that GhTAS sequences may be missed on some assembled chromosomes. The results provide important evidence for the evaluation of the integrity of assembled chromosome end sequences, and will also contribute to the further perfection of the draft genomes of cotton.
\end{abstract}

\section{Keywords}

G. hirsutum, telomere-associated sequence, cloning, FISH

\section{Introduction}

Telomeres are DNA-protein complexes at the ends of chromosomes (Blackburn 1991). Telomere structures are highly conserved, and vary surprisingly little between organisms (Richards and Ausubel 1988, Ganal et al. 1991, Fajkus et al. 2005, Watson and

Copyright Yuling Liu et al. This is an open access article distributed under the terms of the Creative Commons Attribution License (CC BY 4.0), which permits unrestricted use, distribution, and reproduction in any medium, provided the original author and source are credited. 
Riha 2010). In humans, telomere repeated sequences are composed of conserved a minisatellite sequence unit 5'-TTAGGG-3' (Moyzis et al. 1988), whereas in Tetrahymena (Furgason, 1940) each chromosome end has a conserved 5'-TTGGGG-3' telomere repeat unit (Blackburn and Gall 1978). The first plant telomere DNA sequence, 5'-TTTAGGG-3' tandem repeat, was isolated from Arabidopsis thaliana (Linnaeus, 1753) (Richards and Ausubel 1988). Subsequent studies have demonstrated that the Arabidopsis-type telomeres presented in most plants (Fajkus et al. 2005, Ling et al. 2012, Schrumpfová et al. 2019). At the same time, other studies have shown that some plants lacked typical telomere tandem repeat 5'-TTTAGGG-3', which sheds more light on telomere function and how telomeres responded to genetic change (Adams et al. 2001, Sýkorováet al. 2003a, Peška et al. 2015).

Telomere tandem repeats located at the end of chromosomes represent only a part of the end of chromosomes. Telomere-associated sequences (TASs) located directly proximal to telomere tandem repeats ( $\mathrm{Li}$ et al. 2009) play an important role in telomere maintenance and chromosome stability through epigenetic modification or recombination (Cross et al. 1990, Zhong et al. 1998, Sýkorováet al. 2003b, Tran et al. 2015). In addition, TAS is also a good marker at the end of the genetic linkage map. Three TASs cloned from rice showed high polymorphism at the ends of chromosome arms of different rice varieties based on the results of genetic mapping (Ashikawa et al. 1994). Despite functional importance, the nucleotide sequences in the subtelomere region have not been fully resolved in many sequenced genomes (Lese et al. 1999, Mefford and Trask 2002, Mizuno et al. 2006). So, more work is needed to reveal the structure and function of the subtelomeres.

At present, there is relatively little research on cotton telomere. Combining FISH using the Arabidopsis-type telomere sequence amplified from Arabidopsis genomic DNA and BAL-31 digestion, Ling et al. (2012) published the first study on cotton telomeres, which proved the Arabidopsis-type telomere sequence existed in the cotton genome. $G$. hirsutum is the most important cultivated cotton species. So far, different versions of the genome sequence have been released (Li et al. 2015, Zhang et al. 2015, Wang et al. 2019, $\mathrm{Hu}$ et al. 2019), however, high content of repetitive sequences affects the quality of genome assembly (Sýkorová et al. 2013, Liu et al. 2016). TAS occupies a large proportion in subtelomere tandem repeats regions. Therefore, in order to improve the quality of genome assembly, nucleotide sequences in the subtelomere region need to be further analyzed.

\section{Material and methods}

\section{Plant materials}

The plant material was $G$. hirsutum acc. TM-1 (AADD), which was planted in the experimental field of Anyang Institute of Technology, Henan, China. Genomic DNA was isolated from fresh young leaves using the modified CTAB method (Song 
et al. 1998). Root tip material used for G. hirsutum chromosome preparation were harvested from the about 6-day seedlings planted in an incubator and pretreated by $25 \mathrm{ppm}$ cycloheximide at $20{ }^{\circ} \mathrm{C}$ for $80 \mathrm{~min}$, then fixed in methanol-acetic acid (3:1) and stored at $4{ }^{\circ} \mathrm{C}$ for $24 \mathrm{~h}$. Squashes of root tips were prepared according to Liu et al. (2017).

\section{Primers}

The eight single primers of the plant telomere repeat were selected from NCBI database (https://www.ncbi.nlm.nih.gov) according to the previous studies for single primer PCR (Burr et al. 1992, Gong et al. 1998, Weiss-Schneeweiss et al. 2004, Liu et al. 2005). The primers sequence information is shown in Table 1.

\section{Cloning and sequencing of telomere-associated sequences}

The selected single primers of the plant telomere repeat sequence (Table 1) were amplified by single primer PCR using the genomic DNA of $G$. hirsutum as template, to find the suitable conditions for obtaining promising products and candidates for subtelomeric regions. The amplification procedure was as $95^{\circ} \mathrm{C}$ for $3 \mathrm{~min}$, followed by 35 cycles of $95^{\circ} \mathrm{C}$ for $15 \mathrm{~s}, 55^{\circ} \mathrm{C} / 60{ }^{\circ} \mathrm{C}$ for $15 \mathrm{~s}, 72{ }^{\circ} \mathrm{C}$ for $30 \mathrm{~s}$, and a final extension at $72{ }^{\circ} \mathrm{C}$ for $5 \mathrm{~min}$. The amplification products were detected by $1 \%$ agarose gel electrophoresis, and the appropriate single primer and annealing temperature were selected based on the above result. Then, PCR amplification was performed using the selected single primer in a $50 \mu \mathrm{l}$ reaction volume containing $25 \mu \mathrm{l}$ of $2 \times$ Phanta Max Buffer, $1 \mu \mathrm{l}$ of Phanta Max Super-Fidelity DNA Polymerase (Vazyme), $0.8 \mu \mathrm{mol} / \mathrm{L}$ of the telomeric single primer, and $10 \mathrm{ng}$ of genomic DNA. The objective band from PCR was recovered by gel extraction kit (SanPrep Column DNA Gel Extraction kit, Sangon Biotech) and was cloned into Trans1-T1 competent cells by the pEasy-Blunt Simple Cloning Vector (TransGen Biotech) according to the manufacturer's instructions. The positive clones were selected for sequencing by Shanghai Sangon.

Table I. Telomere primer sequence information.

\begin{tabular}{lccc}
\hline Name & Taxonomic name & Reference & Sequence \\
\hline TR1 & Oryza sativa (Linnaeus, 1753) & Gong et al. 1998 & (TTTAGGG) \\
TR2 & Zea mays (Linnaeus, 1753) & Burr et al. 1992 & (TTTAGGG) \\
TR3 & Othocallis siberica (Linnaeus, 1753) & Weiss-Schneeweiss et al. 2004 & (TTTAGGG) \\
TR4 & Ginkgo biloba (Linnaeus, 1771) & Liu et al. 2005 & (CCCTAAA) ${ }_{3}$ \\
TR5 & Brassica campestris (Linnaeus, 1753) & Kapila et al. 1996 & (CCCTAAA) ${ }_{3}$ CCC \\
TR6 & Othocallis siberica & Weiss-Schneeweiss et al. 2004 & AA (CCCTAAA) ${ }_{3}$ CCC \\
TR7 & Zea mays & Burr et al. 1992 & (CCCTAAA) ${ }_{4}$ \\
TR8 & Othocallis siberica & Weiss-Schneeweiss et al. 2004 & (CCCTAAA) ${ }_{5}$ \\
\hline
\end{tabular}




\section{Software and websites for sequences analysis}

DNAMAN software was used for extraction and alignment of cloned sequences. Repetitive sequence analysis was performed using the online program CENSOR (https:// www.girinst.org/censor/index.php). BLAST algorithm blastn (https://www.cottongen. org/blast) was used to identify GhTAS from G. hirsutum genome database (Gossypium hirsutum ZJU v2.1, a1) (Hu et al. 2019). All the above analyses were performed according to the default parameters.

\section{FISH validation}

The TAS plasmid DNA was extracted using the TIANprep Mini Plasmid Kit according to the instructions. Then, TAS plasmid DNA was labeled with DIG-Nick Translation Mix (Roche). The $45 \mathrm{~S}$ rDNA probes derived from Arabidopsis thaliana (Gan et al. 2013) were labeled with biotin-Nick Translation Mix (Roche) according to the instructions of the manufacturer. Chromosome preparation and FISH were performed according to the previous methods (Liu et al. 2017).

\section{Results}

\section{Optimization of the single primer PCR}

According to the melting temperature $(\mathrm{Tm})$ value distribution of the eight candidate single primers $\left(55^{\circ} \mathrm{C}-62{ }^{\circ} \mathrm{C}\right)$, two annealing temperatures were selected, namely $55^{\circ} \mathrm{C}$ and $60{ }^{\circ} \mathrm{C}$. The results of PCR amplification showed that an obvious band of roughly $500 \mathrm{bp}$ was amplified using the single primer TR6 under the two annealing temperatures, especially, the band amplified under annealing temperature of $60^{\circ} \mathrm{C}$ showed better specificity and higher brightness (Fig. 1B-6). So, the primer TR6 (AA (CCCTAAA) ${ }_{3}$ CCC) was chosen for the following PCR amplification.

\section{Cloning of TAS}

A single band with a size of roughly $500 \mathrm{bp}$ was amplified using the single primer TR6 under the annealing temperature of $60{ }^{\circ} \mathrm{C}$ with Phanta Max Super-Fidelity DNA Polymerase (Fig. 2A-2). After transformation, eight positive clones were obtained after a positive test from transformed clones (Fig. 2B). Then, the eight positive clones were sequenced. 


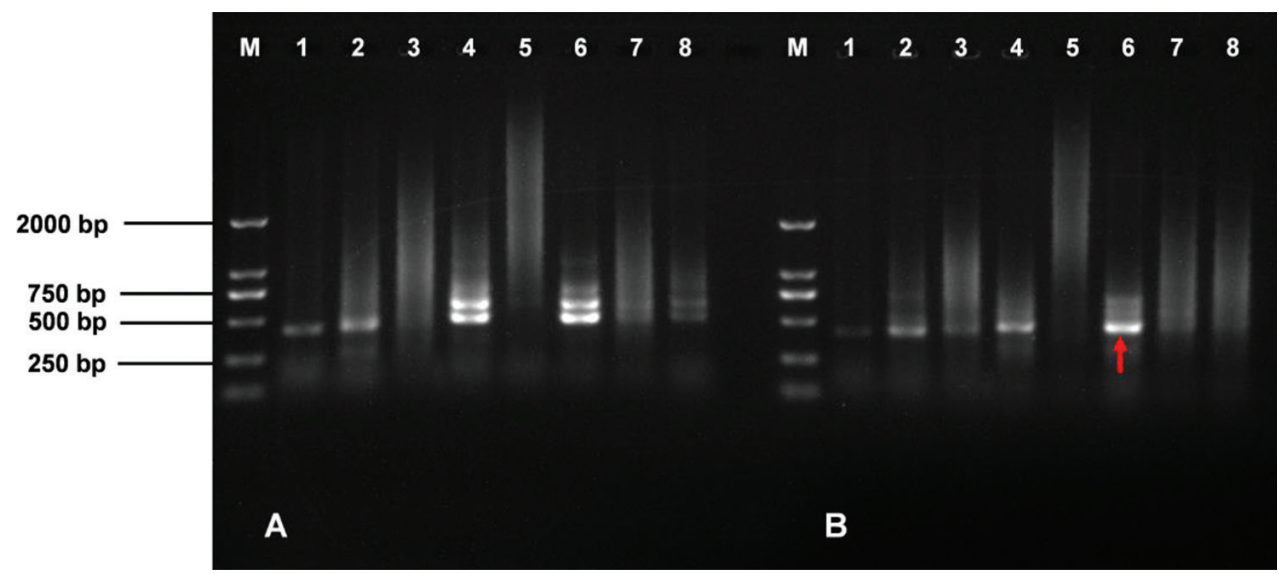

Figure I. Amplification results of candidate single primers. M Marker $\mathbf{A}$ and $\mathbf{B}$ the annealing temperature is 55 and $60{ }^{\circ} \mathrm{C}$ respectively $\mathbf{1 - 8}$ primers TR1-TR8.

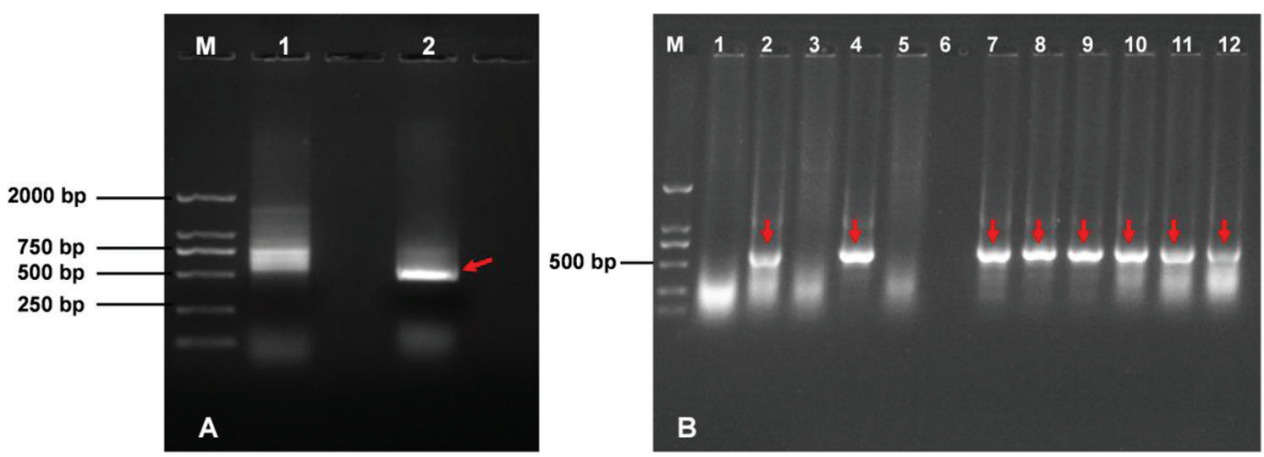

Figure 2. Results of cloning and positive test. A PCR amplification results M Marker $\mathbf{1}$ Common Taq enzyme $\mathbf{2}$ High-fidelity enzyme. B Positive test of bacterial colony PCR M Marker 1-12 the candidate clones.

\section{Sequences analysis}

\section{Sequence component analysis}

Sequence analysis of the eight positive clones revealed that all clones had the same forward and inverted telomere primer sequence at the two ends. Sequence alignment showed that there were two different internal sequences in eight sequences. So, the two different cloned DNA sequences with different size of $488 \mathrm{bp}$ and $538 \mathrm{bp}$ were selected and named as GhTAS1 and GhTAS2 (Fig. 3). Their sequences had been uploaded to GenBank (accession No. MT078976 and MT078977). 


\begin{abstract}
$>$ GhTAS1
AACCCTAAACCCTAAACCCTAAACCCCAAGCACCAAGCCCCAAGCCCTAAAACCTCTAACCTCAGAACCTAAAACCCCAA ACCCTAATACCTTAAACCCCTAAACCTTAAAATCCAAAACTTAATATACATGAATTTGGATATTGATAGAATGAGATATCC CCACCTAGGATATCCAAAATCGGGATATGCCCGAACCGGGATATCCATGGATTAGGATCTCTCGACCGGGGATAAGAGAG GTAGGTTTCCCGGTTCAGAGACATAAATTATCTGAACCGGTCACGAAACTATCCAACCCTGCAGCTAAGATTCTGGACCGG GACAGGGTATGAATGGGATATCCCTGAATTGCTTATGCCAAGCGGGAATAGGAATCGAAAATCCCTGAATTGTTTAGGGT TTGGGTTTTAGAGTTTATGGTTTAAGGTTTTAGGGTTTAAAGTTTAGGATTTATGGTTTAGGGTTTAGGGTTTAGGGTTTAG GGTTTAGGGTT

$>$ GhTAS2

AACCCTAAACCCTAAACCCTAAACCCAGGGATATCCCAATTCACCGTTAACCCATAGTGGTATATCCCGGCCTGGATATAT CACATTCTAGGAATATCCCAATTCCCCACTTTTTTTTTCCTAATTCTCTATACCTTCTTTGTCTTTAATTTCTCAAAAAAAAA AAGTAATTTAAAGAAGGTATTAATTACCATAAATGTTACCGTTGTCATATGGGATTTAGAAAATTCTCTCTCTTCCATTCAT AGCTTCCTCGTTTCTTCACCTATTCATCTACCTCTTTAAATTTATTTTCCTTTTTTATTTCCCCTTTTATTTTCTGTTGTTTTTC CATTCTTTGATCTCTGTTAATCCTCTCTTTTGTTTTCTTTATTCTCTTTCCCTTCCTTTCTTGTCCTTTCG TAAGAAACCCTAGA CACTTCCACGCCATCAACACCTTCCGGCGTCTTACCTCCCATTATTCGAAATTTGAATCCTCAAATCTATCGCTTCTATCCA TACCACCCCATTTAGGGTTTAGGGTTAGGGTTTAGGGTT
\end{abstract}

Figure 3. Sequences of the two TASs. The grey shadows are reverse complementary sequences of the telomere primer TR6.

The two sequences were rich in $\mathrm{A} / \mathrm{T}$ bases, that is, $57.6 \%$ and $63.9 \%$ respectively. Repeat masking analysis indicated that the tandem repeats content were $31.35 \%$ in GhTAS1 and $42.38 \%$ in GhTAS2, which mainly consisted of satellite DNA and transposable elements. The above results are consistent with the typical characteristics of telomere-associated sequences ( $\mathrm{Li}$ et al. 2009).

\title{
Homology analysis of GhTASs
}

Sequence alignment results of DNAMAN shown that GhTAS1 and GhTAS2 had low homology, with the sequence similarity of $38.90 \%$, which may be due to their different chromosomal sources.

After comprehensive comparison of the obtained TASs of G. hirsutum and the TASs of Arabidopsis thaliana, Glycine max (Linnaeus, 1753), Oryza sativa (Linnaeus, 1753), Zea mays (Linnaeus, 1753), Larix gmelinii (Ruprecht, 1920) listed on NCBI, it was found that their similarity was low, ranging from $25 \%$ to $50 \%$ (Table 2). All these indicated that the cloned telomere-associated sequences had obvious species specificity.

\section{BLAST of GhTAS1 and GhTAS2 against G. hirsutum genome}

GhTAS1 and GhTAS2 were found using blastn with the latest G. hirsutum genome sequence (Gossypium hirsutum ZJU v2.1, a1) in Cottongen (https://www.cottongen.org/). 
Table 2. Similarity of telomere-associated sequences between G. hirsutum and other plants.

\begin{tabular}{lccc}
\hline \multicolumn{1}{c}{ Species } & NCBI accession No. & \multicolumn{2}{c}{ TASs of G. hirsutum } \\
& & GhTAS1 & GhTAS2 \\
\hline Arabidopsis thaliana & AC074298.1 & $39.60 \%$ & $36.71 \%$ \\
& AM177016.1 & $14.08 \%$ & $12.94 \%$ \\
& AM177019.1 & $13.52 \%$ & $13.93 \%$ \\
Glycine max & AM177060.1 & $10.88 \%$ & $10.15 \%$ \\
Oryza sativa & AF041999.1 & $20.24 \%$ & $16.79 \%$ \\
Zea mays & $\mathrm{U} 12056.1$ & $28.71 \%$ & $25.27 \%$ \\
Larix gmelinii & S46927.1 & $48.70 \%$ & $41.93 \%$ \\
\hline
\end{tabular}

Table 3. Partial blast results of GhTAS1 and GhTAS2 in the G. hirsutum genome.

\begin{tabular}{|c|c|c|c|c|}
\hline Sequence name & Genomic location & Query matches & Hit matches & Identity (\%) \\
\hline \multirow[t]{6}{*}{ GhTAS1 } & D06 & $1-488$ & $65407147-65406660$ & $98.98 \%$ \\
\hline & D03 & $184-281$ & $26586-26683$ & $78.57 \%$ \\
\hline & A01 & $171-237$ & $118151185-118151119$ & $82.09 \%$ \\
\hline & D02 & $138-219$ & 69751633-69751551 & $79.52 \%$ \\
\hline & Scaffold515-obj & $184-281$ & $9914-9817$ & $75.51 \%$ \\
\hline & D01 & $184-281$ & 64676574-64676477 & $75.51 \%$ \\
\hline \multirow[t]{19}{*}{ GhTAS2 } & A06 & $14-537$ & $126445179-126444656$ & $99.62 \%$ \\
\hline & D11 & $14-537$ & $71336660-71336138$ & $98.47 \%$ \\
\hline & A13 & $14-535$ & 47688-48202 & $94.08 \%$ \\
\hline & A02 & $25-512$ & 40084-39589 & $88.15 \%$ \\
\hline & D02 & $25-512$ & $69751559-69752073$ & $86.68 \%$ \\
\hline & A12 & $25-512$ & $30186-29672$ & $86.15 \%$ \\
\hline & Scaffold546-obj & $46-455$ & $8556-8146$ & $89.07 \%$ \\
\hline & Scaffold515-obj & $25-271$ & $31264-31514$ & $89.33 \%$ \\
\hline & A09 & $25-315$ & $83200103-83200398$ & $86.96 \%$ \\
\hline & A11 & $25-271$ & $121355653-121355904$ & $88.54 \%$ \\
\hline & scaffold407_obj_A03 & $59-271$ & $36503-36719$ & $92.24 \%$ \\
\hline & A07 & $25-271$ & $96580716-96580969$ & $88.24 \%$ \\
\hline & D10 & $278-455$ & 66830830-66831007 & 93.26 \\
\hline & A10 & $25-271$ & $115081227-115081476$ & $87.75 \%$ \\
\hline & A05 & $285-455$ & $39831434-39831267$ & $93.60 \%$ \\
\hline & A01 & $278-455$ & $118169784-118169962$ & $91.06 \%$ \\
\hline & D08 & $278-512$ & 69075939-69076196 & $84.11 \%$ \\
\hline & D03 & $278-455$ & 23313-23139 & $91.01 \%$ \\
\hline & D09 & $278-442$ & 51987281-51987445 & $91.52 \%$ \\
\hline
\end{tabular}

Results showed that GhTAS1 was mapped onto five chromosomes and one scaffold of $G$. hirsutum, and GhTAS2 was mapped onto all 26 chromosomes and 14 scaffolds of $G$. hirsutum with different E-value. The partial blast results with lower E-value were listed in Table 3. GhTAS1 was localized at one end of the chromosome D06, with a higher similarity of $98.98 \%$, and was localized at the single end of chromosomes D03, A01, D02 and D01, as well as Scaffold515, with lower similarity (Fig. 4A). GhTAS2 showed 


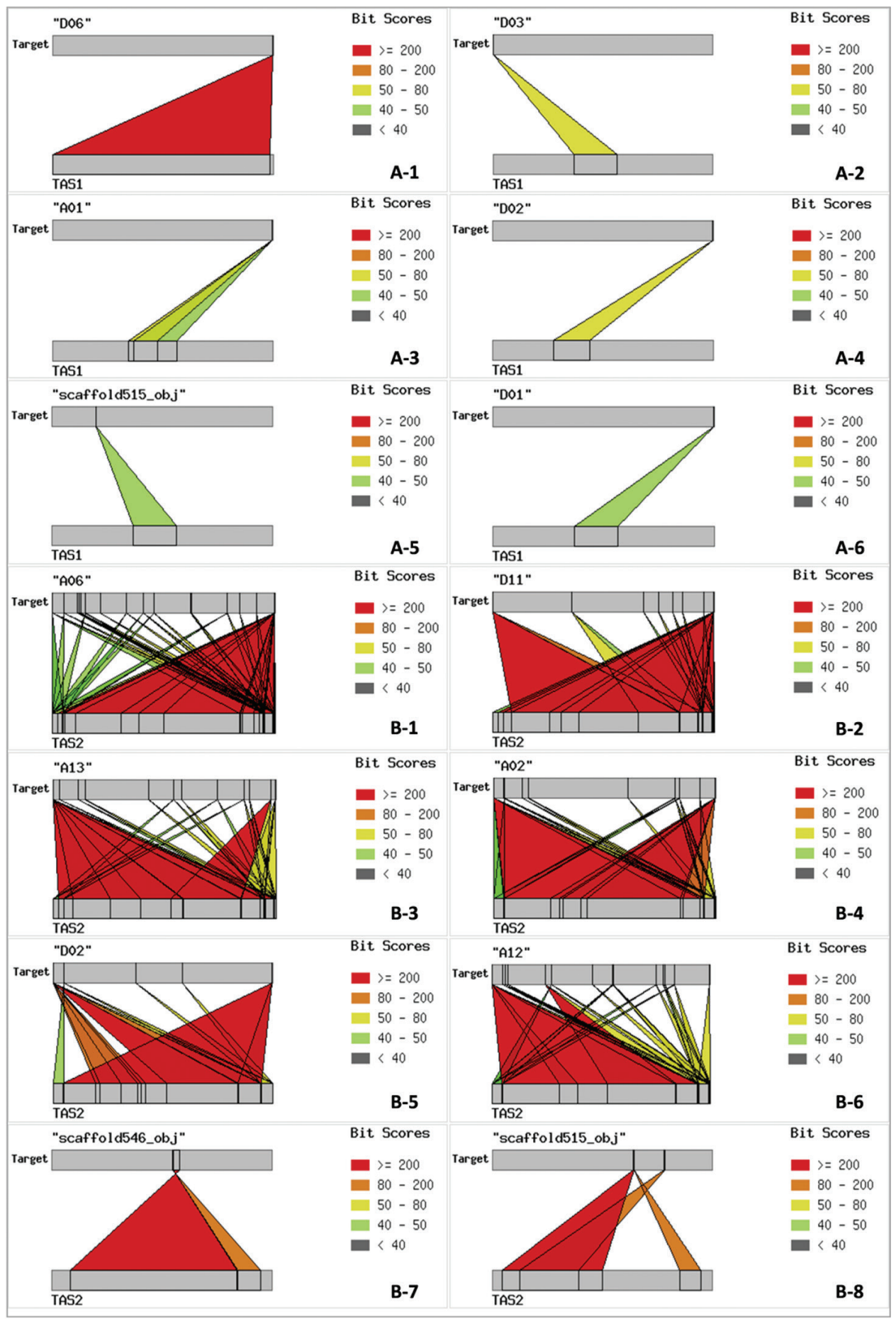

Figure 4. Localization patterns of GhTAS1 and GhTAS2 on G. hirsutum partial chromosomes. A GhTAS1 B GhTAS2. 

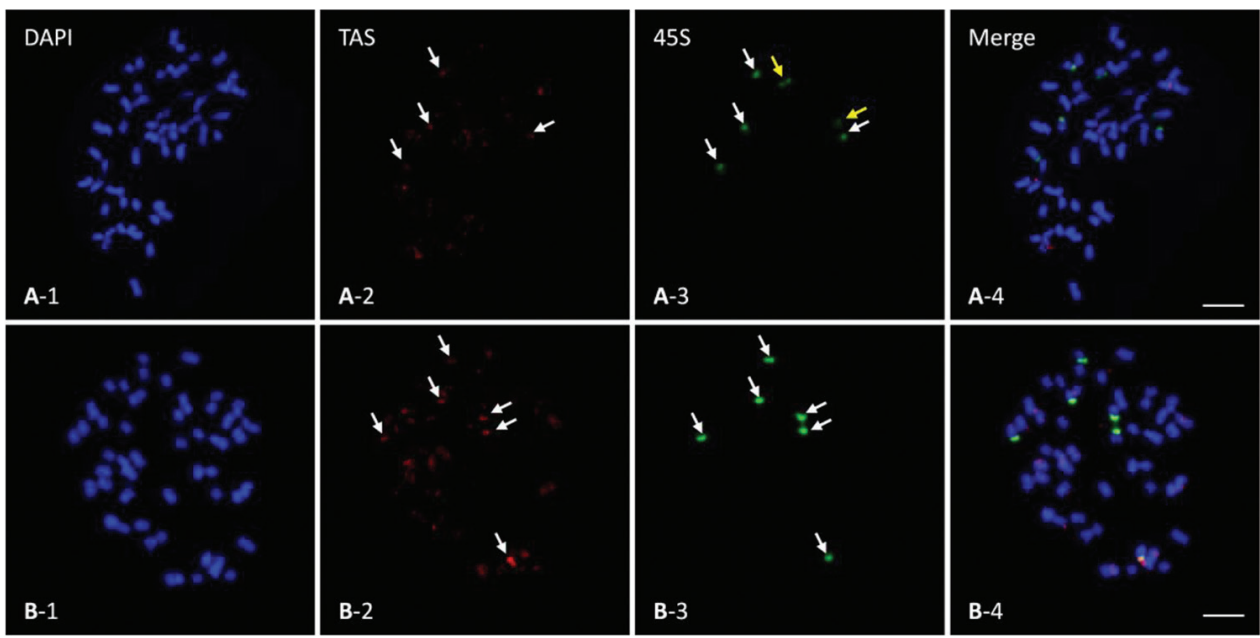

Figure 5. FISH on G. hirsutum chromosome with $45 \mathrm{~S}$ rDNA (green) and GhTAS1 or GhTAS2 (red) probes, Scale bars: $5 \mu \mathrm{m}$. The white arrows showed co-location A GhTAS1 B GhTAS2.

higher chromosomes coverage than GhTAS1. Among the all blast results, GhTAS2 was localized at both ends of chromosomes D11, A13, A02 and D02 and at the single end of chromosomes A06, A12 and two scaffolds with higher similarity (Fig. 4B). At the same time, unlike GhTAS1, the GhTAS2 sequence is also mapped to other chromosomal regions in addition to the ends of chromosomes (Fig. 4B1-6).

\section{Chromosome localization of GhTAS1 and GhTAS2 based on FISH}

To examine the chromosome physical location of GhTAS1 and GhTAS2, we carried out FISH on $G$. hirsutum metaphase chromosomes using a digoxin-labeled GhTAS probe and a biotion-labeled $45 \mathrm{~S}$ rDNA probe. The results showed that GhTAS1 had signals at the end of nearly half of the chromosomes of G. hirsutum, and most of them were distributed at the single end. The signal intensity on different chromosomes was also different (Fig. 5A-2, A-4). GhTAS2 has signals on both ends of most chromosomes of G. hirsutum (Fig. 5B-2, B-4). Three pairs of $45 \mathrm{~S}$ rDNA signals were detected on the chromosomes of G. hirsutum (Fig. 5a-3 and 5B-3 arrows). Two pairs of GhTAS1 signals were collinear with $45 \mathrm{~S}$ rDNA (Fig. 5A-2 arrows). Three pairs of GhTAS2 signals were collinear with $45 \mathrm{~S}$ rDNA (Fig. 5B-2 arrows). In addition, the chromosomes carrying GhTAS2 FISH signals were much more than those with GhTAS1 FISH signals (Fig. 5A-2, B-2), which was similar to the blast results (Fig. 4, Table 3). 


\section{Discussion}

In this study, the telomere primer $\mathrm{AA}(\mathrm{CCCTAAA})_{3} \mathrm{CCC}$ was used as a single primer to obtain the TAS sequences of $G$. hirsutum by single primer PCR. The homology of the two TASs is relatively low and with the similarity of $38.90 \%$. Chromosome FISH localization of the two sequences also showed obvious differences in chromosome distribution and signal strength (Fig. 5A, B), which may be due to the differences of chromosome specificity and sequence copy number of the two TASs. In the early study of Chironomus palidivittatus (Edwards, 1929) TAS, it was found that there were considerable differences in TAS between species, within species, and even in telomere of the same species (Cohn and Edstrom 1992). Gong et al. cloned six TASs in rice and found high polymorphism of these sequences through RFLP analysis (Gong et al. 1998). From then on, this phenomenon has been found in related studies of other species (Li et al. 2009). Therefore, TASs show great specificity, unlike the more conservative telomere repeated sequences (TR).

Since telomere and adjacent subtelomere regions could not be covered by PAC and $\mathrm{BAC}$ clones, sequencing efforts were unable to reveal the structure of these regions. In addition, the discovery of interstitial telomeric sequences (ITSs) makes telomeric minisatellites have double-faced character, which causes more problems in producing genomic assemblies (Richards et al. 1991, Sýkorová et al. 2003). Therefore, nucleotide sequences in the subtelomere regions have not been fully resolved in many genomes that have been sequenced (Mefford and Trask 2002, Mizuno et al. 2006), which greatly affects the quality of genome assembly. FISH localization can reflect the true position of DNA fragments in chromosomes (Jiang and Gill 2006). FISH combined with genomic BLAST can intuitively judge the genomic assembly quality of DNA sequences. Chromosomal locations of $45 \mathrm{~S} \mathrm{rDNA}$ in $G$. hirsutum had been revealed using double-probe FISH, that is, chromosomes A09, D07 and D09 (Gan et al. 2013). In this study, according to the genome BLAST and chromosome FISH localization results of GhTAS and $45 \mathrm{~S}$ rDNA, it was found that TASs at the end of some chromosomes were not assembled in the genome sequence map. Obviously, results of blastn showed that GhTAS1 was only mapped onto chromosomes D06, D03, A01, D02 and D01 (Table 3, Fig. 4A), but FISH showed more chromosomes carried GhTAS1 signals, including two of the three chromosomes with $45 \mathrm{~S}$ rDNA A09, D07 or D09, which had not appeared on the blastn results. That is, GhTAS1 sequences may be missed on these assembled chromosomes. The results provide important evidence for the evaluation of the integrity of assembled chromosome end sequences.

\section{Conclusions}

We cloned two telomere-associated sequences from $G$. hirsutum acc. TM-1 using the single-primer PCR, and made analysis about the sequence characteristics of two TASs. The two TASs sequences were enriched in $\mathrm{A} / \mathrm{T}$, and were flanked by the forward and in- 
verted primer sequences at each end respectively. By comparative analysis based on the results of blastn and FISH localization of the two TASs, we found that TASs at the end of some chromosomes were not assembled in the genome sequence map. Our study not only contributes to the analysis of telomere structure of cotton, but also provides intuitive evidence for the evaluation of the integrity of the assembled $G$. hirsutum genome.

\section{Acknowledgements}

The research was sponsored by National Key R\&D Program of China (No. 2018YFD0100300), Innovation Scientists and Technicians Troop Construction Projects of Henan Province (20IRTSTHN021), Science and Technology Development Program of Anyang City (2018-66-133), Science and Technology Development Project of Henan Province (182102410041).

\section{References}

Adams SP, Hartman TPV, Lim KY, Chase MW, Bennett MD, Leitch IJ, Leitch AR (2001) Loss and recovery of Arabidopsis-type telomere repeat sequences 5'-(TTTAGGG)n-3' in the evolution of a major radiation of flowering plants. Proceedings of the Royal Society B: Biological Sciences 268(1476): 1541-1546. https://doi.org/10.1098/rspb.2001.1726

Ashikawa I, Kurata N, Nagamura Y, Minobe Y (1994) Cloning and mapping of telomere-associated sequences from rice. DNA Research 1: 67-76. https://doi.org/10.1093/dnares/1.2.67

Blackburn EH (1991) Structure and function of telomeres. Nature 350: 569-573. https://doi. org/10.1038/350569a0

Blackburn EH, Gall JG (1978) A tandemly repeated sequence at the termini of the extrachromosomal ribosomal RNA genes in Tetrahymena. Journal of Molecular Biology 120(1): 33-53. https://doi.org/10.1016/0022-2836(78)90294-2

Burr B, Burr FA, Matz EC, Romeroseverson J (1992) Pinning down loose ends: mapping telomeres and factors affecting their length. Plant Cell 4: 953-960. https://doi.org/10.1105/tpc.4.8.953

Cohn M, Edstrom JE (1992) Chromosome ends in Chironomus pallidivittatus contain different subfamilies of telomere-associated sequences. Chromosoma 101(10): 634-640. https:// doi.org/10.1007/BF00360541

Cross S, Lindsey J, Fantes J, McKay S, McGill N, Cooke H (1990) The structure of a subterminal repeated sequence present on many human chromosomes. Nucleic Acids Research 18(22): 6649-6657. https://doi.org/10.1093/nar/18.22.6649

Fajkus J, Sýkorová E, Leitch AR (2005) Telomeres in evolution and evolution of telomeres. Chromosome Research 13: 469-479. https://doi.org/10.1007/s10577-005-0997-2

Gan YM, Liu F, Chen D, Wu Q, Qin Q, Wang CY, Li SH, Zhang XD, Wang YH, Wang KB (2013) Chromosomal locations of $5 \mathrm{~S}$ and $45 \mathrm{~S}$ rDNA in Gossypium genus and its phylogenetic implications revealed by FISH. PLoS ONE 8(6): e68207. https://doi.org/10.1371/ journal.pone.0068207 
Ganal MW, Lapitan NL, Tanksley SD (1991) Macrostructure of the tomato telomeres. Plant Cell 3: 87-94. https://doi.org/10.1105/tpc.3.1.87

Gong XQ, Gong JM, Liu F, Chen SY (1998) Screening and localization of BAC clones containing telomere-associated sequences in rice. Science in China (Series C) 28: 437-443. [In Chinese]

Hu Y, Chen J, Fang L, Zhang Z, Ma W, Niu Y et al. (2019) Gossypium barbadense and Gossypium hirsutum genomes provide insights into the origin and evolution of allotetraploid cotton. Nature Genetics 51(4): 739-748. https://doi.org/10.1038/s41588-019-0371-5

Jiang JM, Gill BS (2006) Current status and the future of fluorescence in situ hybridization (FISH) in plant genome research. Genome 49(9): 1057-1068. https://doi.org/10.1139/g06-076

Kapila R, Das S, Lakshmikumaran M, Srivastava PS (1996) A novel species-specific tandem repeat DNA family from Sinapis arvensis: detection of telomere-like sequences. Genome 39(4): 758-766. https://doi.org/10.1139/g96-095

Lese CM, Fantes JA, Riethman HC, Ledbetter DH (1999) Characterization of physical gap sizes at human telomeres. Genome Research 9: 888-894. https://doi.org/10.1101/gr.9.9.888

Li F, Fan G, Lu C, Xiao G, Zou C, Kohel RJ et al. (2015) Genome sequence of cultivated Upland cotton (Gossypium hirsutum TM-1) provides insights into genome evolution. Nature Biotechnology 33: 524-530. https://doi.org/10.1038/nbt.3208

Li J, Yang F, Zhu J, He SB, Li LJ (2009) Characterization of a tandemly repeated subtelomeric sequence with inverted telomere repeats in maize. Genome 52(3): 286-293. https://doi. org/10.1139/G09-005

Ling J, Cheng H, Liu F, Song GL, Wang CY, Li SH, Zhang XD, Wang YH, Wang KB (2012) The cloning and fluorescence in situ hybridization analysis of cotton telomere Sequence. Journal of Integrative Agriculture 11(9): 1417-1423. https://doi.org/10.1016/S2095-3119(12)60141-5

Liu D, Lu H, Ji FT, Li FL, Guo HH (2005) Cloning and Analysis of telomere-associated Sequences of Ginkgo biloba L. Forestry Studies in China 7(1): 7-10. https://doi.org/10.1007/ s11632-005-0049-1

Liu YL, Liu Z, Peng RH, Wang YH, Zhou ZL, Cai XY, Wang XX, Zhang ZM, Wang KB, Liu F (2017) Cytogenetic maps of homoeologous chromosomes Ah01 and Dh01 and their integration with the genome assembly in Gossypium hirsutum. Comparative Cytogenetics 11(2): 405-420. https://doi.org/10.3897/CompCytogen.v11i2.12824

Liu YL, Peng RH, Liu F, Wang XX, Cui XL, Zhou ZL, Wang CY, Cai XY, Wang YH, Lin ZX, Wang KB (2016) A Gossypium BAC clone contains key repeat components distinguishing sub-genome of allotetraploidy cottons. Molecular Cytogenetics 9(1): 27. https://doi. org/10.1186/s13039-016-0235-y

Mefford HC, Trask BJ (2002) The complex structure and dynamic evolution of human subtelomeres. Nature Reviews Genetics 3(2): 91-102. https://doi.org/10.1038/nrg727

Mizuno H, Wu J, Kanamori H, Fujisawa M, Namiki N, Saji S, Katagiri S, Katayose Y, Sasaki T, Matsumoto T (2006) Sequencing and characterization of telomere and subtelomere regions on rice chromosomes $1 \mathrm{~S}, 2 \mathrm{~S}, 2 \mathrm{~L}, 6 \mathrm{~L}, 7 \mathrm{~S}, 7 \mathrm{~L}$ and $8 \mathrm{~S}$. The Plant Journal 46(2): 206-217. https://doi.org/10.1111/j.1365-313X.2006.02684.x

Moyzis RK, Buckingham JM, Cram LS, Dani M, Deaven LL, Jones MD, Meyne J, Ratliff RL, Wu JR (1988) A highly conserved repetitive DNA sequence, (TTAGGG)n, present at the telomeres of human chromosomes. Proceedings of National Academy of Sciences, United States 85(18): 6622-6626. https://doi.org/10.1073/pnas.85.18.6622 
Peška V, Fajkus P, Fojtová M, Dvořáčková M, Hapala J, Dvořáček V, Polanská P, Leitch AR, Sýkorová E, Fajkus J (2015) Characterisation of an unusual telomere motif (TTTTTTAGGG) $\mathrm{n}$ in the plant Cestrum elegans (Solanaceae), a species with a large genome. The Plant Journal 82(4): 644-654. https://doi.org/10.1111/tpj.12839

Richards EJ, Ausubel FM (1988) Isolation of a higher euikaryotic telomere from Arabidopsis thaliana. Cell 53: 127-136. https://doi.org/10.1016/0092-8674(88)90494-1

Richards EJ, Goodman HM, Ausubel FM (1991) The centromere region of Arabidopsis thaliana chromosome 1 contains telomere-similar sequences, Nucleic Acids Research 19: 3351-3357. https://doi.org/10.1093/nar/19.12.3351

Schrumpfová P, Fojtová M, Fajkus J (2019) Telomeres in plants and pumans: not so different, not so similar. Cells 8(1): 58. https://doi.org/10.3390/cells8010058

Song GL, Cui RX, Wang KB, Guo LP, Li SH, Wang CY, Zhang XD (1998) A rapid improved CTAB method for extraction of cotton genomic DNA. Cotton Science 10(5): 273-275. [In Chinese]

Sýkorová E, Cartagena J, Horakova M, Fukui K, Fajkus J (2003a) Characterization of telomeresubtelomere junctions in Silene latifolia. Molecular Genetics Genomics 269(1): 13-20. https://doi.org/10.1007/s00438-003-0811-9

Sýkorová E, Fojtová M, Peška V (2013) A PCR-based approach for evaluation of telomere associated sequences and interstitial telomeric sequences. Analytical Biochemistry 439(1): 8-10. https://doi.org/10.1016/j.ab.2013.03.034

Sýkorová E, Lim KY, Chase MW, Knapp S, Leitch IJ, Leitch AR, Fajkus J (2003b) The absence of Arabidopsis-type telomeres in Cestrum and closely related genera Vestia and Sessea (Solanaceae): first evidence from eudicots. The Plant Journal 34(3): 283-291. https://doi. org/10.1046/j.1365-313X.2003.01731.x

Tran TD, Cao HX, Jovtchev G, Neumann P, Novák P, Fojtová M, Vu GTH, Macas J, Fajkus J, Schubert I, Fuchs J (2015) Centromere and telomere sequence alterations reflect the rapid genome evolution within the carnivorous plant genus Genlisea. The Plant Journal 84(46): 1087-1099. https://doi.org/10.1111/tpj.13058

Wang MJ, Tu LL, Yuan DJ, Zhu D, Shen C, Li JY et al. (2019) Reference genome sequences of two cultivated allotetraploid cottons, Gossypium hirsutum and Gossypium barbadense. Nature Genetics 51(2): 224-229. https://doi.org/10.1038/s41588-018-0282-x

Watson JM, Riha K (2010) Comparative biology of telomeres: where plants stand. FEBS Letters 584(17): 3752-3759. https://doi.org/10.1016/j.febslet.2010.06.017

Weiss-Schneeweiss H, Riha K, Jang CG, Puizina J, Scherthan H, Schweizer D (2004) Chromosome termini of the monocot plant Othocallis siberica are maintained by telomerase, which specifically synthesizes vertebrate-type telomere sequences. The Plant Journal 37: 484-493. https://doi.org/10.1046/j.1365-313X.2003.01974.x

Zhang TZ, Hu Y, Jiang WK, Fang L, Guan XY, Chen JD et al. (2015) Sequencing of allotetraploid cotton (Gossypium hirsutum L. acc. TM-1) provides a resource for fiber improvement. Nature Biotechnology 33(5): 531-537. https://doi.org/10.1038/nbt.3207

Zhong XB, Fransz PF, Wennekes-van Eden J, Ramanna MS, van Kammen A, Zabel P, de Jong JH (1998) FISH studies reveal the molecular and chromosomal organization of individual telomere domains in tomato. The Plant Journal 13(4): 507-517. https://doi.org/10.1046/ j.1365-313X.1998.00055.x 